\title{
Transcranial magnetic stimulation over dorsolateral prefrontal cortex in Parkinson's disease
}

\author{
Miguel Fernández del Olmo, Olalla Bello, Javier Cudeiro
}

\begin{abstract}
Objective: Several studies have shown that repetitive transcranial magnetic stimulation (rTMS) over the dorsolateral prefrontal cortex (DLPFC) is effective in the treatment of depression in patients with Parkinson disease (PD). However, since research into the effect of this type of rTMS regime on motor function is limited, we studied the effect of rTMS over the DLPFC on the motor functions in PD patients.

Methods: Thirteen patients were randomly assigned into 2 groups, one receiving real-rTMS (90\% of resting motor threshold, $10 \mathrm{~Hz}, 450$ pulses-day for 10 consecutive days) over the DLPFC contralateral to the more affected side, and the other group receiving sham-rTMS. Assessment included a clinical motor evaluation using part III of the Unified Parkinson's Disease Rating Scale (UPDRS), and several motor tasks. The UPDRS was applied before and after 10 days of rTMS. Finger tapping, reach movement, grip movement and gait were measured in each session before and after the rTMS over the 10 day period.

Results: Statistical analysis (ANOVA for repeated measures; group * day * side * rTMS) only showed a significant effect for finger tapping, reach movement and gait for the factor day. No significant change was reported for the UPDRS in any group.

Conclusions: Application of rTMS over the DLPFC as a 10 day course had no significant effect on motor functions and clinical motor status, and the improvement in performance of motor tasks can be attributed to the effects of practice.

Significance: rTMS over the DLPFC did not lead to any motor improvement in PD patients.
\end{abstract}

Keywords: rTMS; Gait; Parkinson; Finger tapping; Reaching; Prefrontal cortex

\section{Introduction}

Transcranial magnetic stimulation (TMS), a non-invasive means of stimulating neurons in the human cerebral cortex, is able to modify neuronal activity locally and at distant sites when delivered in series or trains of pulses. Previous studies have demonstrated the potential modulatory effects of repetitive transcranial magnetic stimulation (rTMS) on the excitability of cortical neurons (Maeda et al., 2000 and Pascual-Leone et al., 1994a) and this effect depends on the parameters used in the stimulation such as intensity, frequency, site of stimulation, and can last beyond the duration of the rTMS (PascualLeone et al., 1998, Chen et al., 1997 and Touge et al., 2001). The mechanisms of the modulation of cortical excitability beyond the duration of the rTMS train are still unclear but animal studies have suggested that modulation of neurotransmitters and gene induction may contribute to these long-lasting modulatory effects (Schlaepfer and Rupp, 2002). In humans different studies have shown changes in cerebral blood flow and glucose metabolism induced by transcranial magnetic stimulation (Fox et al., 1997 and Siebner et al., 1998). These findings raise the possibility of therapeutic applications in neurological and psychiatric disorders.

It is well known that the patients with Parkinson's disease (PD) show several disturbances in their movement, particularity in the gait and in the repetitive and rhythmic upper limb movements (Morris et al., 1994, Morris et al., 1996 and Nakamura et al., 1978). Pascual-Leone et al., 1994b and Pascual-Leone et al., 1994c reported an improvement in motor performance during $5 \mathrm{~Hz}$ rTMS to the motor cortex, in patients with PD. However, a real efficacy of rTMS on motor cortex in PD is controversial since subsequent studies show contradictory results (using figure-of-eight coil: Ghabra et al., 1999, Siebner et 
al., 2000, Khedr et al., 2003, Lefaucheur et al., 2004 and Khedr et al., 2006. Using circular coil: Tergau et al., 1999, Shimamoto et al., 2001, Ikeguchi et al., 2003, Okabe et al., 2003 and Mally et al., 2004). These different results could be due to the different methodologies used in the TMS stimulation such as the shape of coil, the stimulus intensity, frequency and number of pulses (Fregni et al., 2005).

The rTMS over the dorsolateral prefrontal cortex (DLPFC) has been established as a less invasive alternative to electroconvulsive therapy for the treatment of depression (Paus and Barret, 2004) and recent studies show that the rTMS over DLPFC has the same antidepressant efficacy as fluoxetine in patients with PD and concurrent depression (Fregni et al., 2004 and Boggio et al., 2005) and may have additional advantage of some motor improvement with fewer adverse effects (Fregni et al., 2004). It has been reported that successive low frequency $(0.2 \mathrm{~Hz})$ rTMS over frontal cortex (F3-F4 of the international 1020 system) at $70 \%$ of the maximal stimulator output improves several kinds of movements in patients with PD (Ikeguchi et al., 2003). On other hand, in normal subjects the application of several trains of $10 \mathrm{~Hz}$ rTMS over the left dorsolateral prefrontal cortex (DLPFC) with a circular coil caused a reduction in [11C]raclopride binding in the left dorsal caudate nucleus and induced the release of endogenous dopamine in the ipsilateral caudate nucleus (Strafella et al., 2001). Recently, Lomarev et al. (2005) showed a therapeutic effect of high frequency rTMS effect on the motor function in patients with PD after 8 sessions of rTMS applied over the left and right motor cortex and DLPFC. However, since they used several cortical targets simultaneously, the effect of high frequency rTMS over the DLPFC on the motor performance in PD patients is still unknown.

Based on the findings described above, we planned to evaluate the effect of high frequency $(10 \mathrm{~Hz})$ of rTMS over dorsolateral prefrontal cortex in the motor functions in a group of patients with PD, using the protocol of stimulation described previously by Strafella et al. (2001) for a period of 10 consecutive days.

\section{Materials and methods}

\subsection{Subjects}

Thirteen patients with Parkinson's disease were recruited for this study, 6 male and 7 female, aged 61.7 (SD 5.22). All patients provided informed consent according to the declaration of Helsinki (1964). PD subjects were excluded if they had musculoskeletal, cardiovascular or visual disturbances that affected walking ability. A fundamental requirement for inclusion in the study was the ability to walk up and down a $30 \mathrm{~m}$ walkway five times without walking aids or assistance. For PD subjects the level of functional disability was also determined by mean of the motor section (part III) of the Unified Parkinson's Disease Rating Scale (UPDRS) and Hoehn and Yahr scales. No patient showed dementia as assessed by mini-mental state examination (MMSE), freezing episodes nor therapy wash-out. The clinical details of the patients are shown in Table 1. Drug treatment was kept unchanged throughout the study. The Ethics Committee of Our Institution approved the experimental protocol.

\subsection{General procedure}

The patients were randomly allocated to either a real-rTMS condition $(n=8)$ and sham-rTMS condition $(n=5)$. In the first session (D0) we determined the resting motor threshold for rTMS for all patients. Over the next two sessions subjects were trained in the motor task to limit any effects of learning. In a separate session (T1) the results in the motor performance and the UPDRS were recorded. After this the patients performed the motor tasks before and after receiving the rTMS, for 10 days (Days 1-10). One day after the last session with the rTMS the UPDRS was recorded again (T2). Considering the daily fluctuation of parkinsonian symptoms, we carried out sessions at the same time of the day. Patients were in "ON" state through out each session.

\subsection{Magnetic stimulation}

All experiments were performed using a focal stimulating coil, figure-of-8-shaped coil (external diameter of $9 \mathrm{~cm}$ ) powered from a MagstimRapid device (The Magstim Company, Whiyland, UK). The coil was oriented so that it induced electric currents in the brain that flowed in a posterior-to-anterior direction over the hand area of motor cortex. The motor evoked potentials (MEPs) were recorded from the contralateral first dorsal interosseus (FDI) with Ag/Cl surface electrodes fixed on the skin with a belly tendon montage. Threshold was determined for the relaxed FDI. Threshold was defined as the lowest intensity required eliciting a clear response of more than $50 \mu \mathrm{V}$ peak-to-peak amplitude at least $50 \%$ of successive trials (Rossini et al., 1994). The location for the rTMS stimulation in the prefrontal 
dorsolateral cortex (DLPFC) was determined $(5 \mathrm{~cm}$ anterior in the same parasagittal plane as to the optimal scalp position for activation of FDI) (Pascual-Leone et al., 1996). The coil was centered over this position and the handle of the coil was placed posterior and lateral with respect to the midsagittal line.

During the frontal real-rTMS condition, three rTMS blocks were delivered over the contralateral DLPFC to the more affected side. Each block consisted of 150 pulses distributed in 15 trains delivered at $10 \mathrm{~Hz}$ with 1-s duration and $10 \mathrm{~s}$ interval between trains. Each block was separated by 10 min interval. Thus, a total of 450 stimuli were delivered over a period of $30 \mathrm{~min}$. The intensity utilized was set at $90 \%$ of the resting motor threshold (RMT).

During frontal sham-rTMs condition, the center of the magnetic coil was positioned $7 \mathrm{~cm}$ rostrally to the vertex over the midline with the coil angled at $45^{\circ}$ with only the edge of the coil resting on the scalp (Siebner et al., 2000). The protocol utilized in the sham-rTMS condition was identical to the real-rTMS condition.

Table 1. Clinical characteristics of patients with Parkinson disease

\begin{tabular}{|c|c|c|c|c|c|c|c|c|}
\hline $\begin{array}{l}\text { Case } \\
\text { No }\end{array}$ & $\begin{array}{l}\text { Age } \\
\text { (years) }\end{array}$ & Sex & $\begin{array}{l}\text { Disease } \\
\text { duration } \\
\text { (years) }\end{array}$ & $\begin{array}{l}\text { More } \\
\text { affected } \\
\text { side }\end{array}$ & Handedness & $\begin{array}{l}\text { Predominant } \\
\text { symptoms }\end{array}$ & $\mathrm{H} \& \mathrm{Y}^{\mathrm{a}}$ & Medication per day (mg) \\
\hline 1 & 66 & M & 5 & $\mathrm{R}$ & $\mathrm{R}$ & Rigidity/Bradykinesia & 2 & $\begin{array}{l}\text { Levodopa/Carbidopa } \\
\text { 500/125, Entacapone 600, } \\
\text { Pramipexole } 2.1\end{array}$ \\
\hline 2 & 56 & $\mathrm{~F}$ & 2 & $\mathrm{~L}$ & $\mathrm{R}$ & Tremor & 2 & $\begin{array}{l}\text { Levodopa/Carbidopa 200/50, } \\
\text { Cabergoline } 4 \\
\text { Levodopa/Carbidopa }\end{array}$ \\
\hline 3 & 55 & $\mathrm{~F}$ & 12 & $\mathrm{R}$ & $\mathrm{R}$ & Rigidity/Bradykinesia & 2 & $\begin{array}{l}\text { 500/125, Biperiden 4, } \\
\text { Entacapone 200, Pergolide } \\
\text { Mesylate } 1\end{array}$ \\
\hline 4 & 72 & M & 11 & $\mathrm{R}$ & $\mathrm{R}$ & Tremor & 2 & $\begin{array}{l}\text { Levodopa/Carbidopa } \\
\text { 600/150, Pergolide Mesylate } \\
\text { 3, Selegiline } 10\end{array}$ \\
\hline 5 & 54 & M & 1 & $\mathrm{R}$ & $\mathrm{R}$ & Tremor & 1 & $\begin{array}{l}\text { Pramipexole } 2.1 \\
\text { Levodopa/Benserazide }\end{array}$ \\
\hline 6 & 56 & M & 11 & $\mathrm{~L}$ & $\mathrm{R}$ & Rigidity/Bradykinesia & 2 & $\begin{array}{l}\text { 400/100, Cabergoline 2, } \\
\text { Selegiline } 10\end{array}$ \\
\hline 7 & 70 & $\mathrm{~F}$ & 8 & $\mathrm{R}$ & R-L & Tremor & 3 & $\begin{array}{l}\text { Levodopa/Carbidopa } \\
\text { 400/100, } \\
\text { Levodopa/Benserazide } \\
\text { 250/75, Pramipexole 1.05 } \\
\text { Levodopa/Benserazida }\end{array}$ \\
\hline 8 & 70 & M & 10 & $\mathrm{~L}$ & $\mathrm{R}$ & Tremor & 2 & $\begin{array}{l}\text { 450/112.5, Entacapona 800, } \\
\text { Pramipexole 2.1, Selegiline } \\
10\end{array}$ \\
\hline 9 & 72 & $\mathrm{~F}$ & 7 & $\mathrm{R}$ & $\mathrm{R}$ & Tremor & 3 & $\begin{array}{l}\text { Levodopa/Carbidopa 300/75, } \\
\text { Levodopa/Benserazide } \\
\text { 1000/250, Entacapone } 800\end{array}$ \\
\hline 10 & 71 & $\mathrm{~F}$ & 7 & $\mathrm{R}$ & $\mathrm{R}$ & Rigidity/Bradykinesia & 2 & $\begin{array}{l}\text { Levodopa/Benserazide } \\
\text { 600/150, Pramipexole } 2.1\end{array}$ \\
\hline 11 & 66 & M & 3 & $\mathrm{R}$ & $\mathrm{R}$ & Rigidity/Bradykinesia & 2 & $\begin{array}{l}\text { Pramipexole 2.1, Selegiline } \\
10 \\
\text { Levodopa/Carbidopa }\end{array}$ \\
\hline 12 & 74 & $\mathrm{~F}$ & 21 & $\mathrm{R}$ & $\mathrm{R}$ & Tremor & 3 & $\begin{array}{l}\text { 400/100, } \\
\text { Levodopa/Benserazide } \\
\text { 300/75, Pramipexole } 1.05\end{array}$ \\
\hline 13 & 62 & $\mathrm{~F}$ & 7 & $\mathrm{R}$ & $\mathrm{R}$ & Rigidity/Bradykinesia & 2 & $\begin{array}{l}\text { Levodopa/Carbidopa 500/50, } \\
\text { Biperiden 4, Cabergoline } 2\end{array}$ \\
\hline
\end{tabular}

a Hoehn and Yahr Rating Scale. 


\subsection{Motor tasks}

\subsubsection{Finger tapping}

All participants performed repetitive flexion-extension movements with their index finger in two different conditions:

(a) at preferred speed. Subjects were instructed to tap at their preferred diadochokinesis rate for a period of $30 \mathrm{~s}$.

(b) at maximum speed. Subjects were instructed to tap "as quickly as possible” for a period of $10 \mathrm{~s}$.

Tapping was performed separately with index finger on both sides separately. A flexible metal loop was fitted snugly to the index finger just proximal to the terminal interphalangeal joint. Each time the metal loop on the index finger struck the contact plate it completed an electrical circuit and generated a brief voltage pulse. Subjects were seated with their forearms resting on a support (elbow angle about $90^{\circ}$ ). To prevent wrist flexion/extension, the wrist was immobilised by a cast. This procedure assured that the sequencing task could be accomplished only by a tapping motion of the index finger. The dependent variables that were measured for each condition of finger tapping included the tapping frequency in $\mathrm{Hz}$ and the coefficient of variation (CV) of interval of two consecutive taps (in ms) as a indicator of temporal variability of tapping, where $\mathrm{CV}=($ standard deviation/mean $) \times 100$.

\subsubsection{Grip}

Maximum isometric grip strength was measured using a standard hand dynamometer with the grip span fixed at $6 \mathrm{~cm}$. To perform these trials, subjects gripped the dynamometer between the index finger and thumb, and then they increased the grip force as quickly as possible for $5 \mathrm{~s}$. The grip strength was measured for each hand separately. The dependent variables that were measured included the maximum force isometric and the maximum slope.

\subsubsection{Reaching}

All participants performed reaching movements with one arm stretched to touch a point situated in front of them in two different conditions:

(a) At preferred speed. Subjects were instructed to reach and touch a point at their preferred diadochokinesis. Ten consecutives trials were collected.

(b) At maximum speed. Subjects were instructed to reach and touch "as fast as possible" in response to an auditory "go" tone presented randomly every 5-15 s. Five consecutives trials were collected.

Reaching movements were performed with each arm separately. An accelerometer was placed in the wrist. The dependent variables that were measured included the coefficient of variation of velocity in the movement at preferred diadochokinesis and the maximum velocity in the movement at maximum speed.

\subsubsection{Gait}

All participants walked at preferred and maximum speed, back and forth over a $7 \mathrm{~m}$ flat walkway to complete a total distance of $28 \mathrm{~m}$ (with three turns). Two pairs of photoelectric cells, one at the beginning and one at the end of the walkway, were used to measure the time required to complete the task.

\subsection{Analysis}

To test whether the real and sham rTMS groups had similar motor performance we used Student's $t$ test for independent samples. Two separate analyses were performed in order to establish the effect of the rTMS on the clinical status and motor performance. First, two-tailed paired Student's $t$-test were performed to investigate changes in the clinical status before (T1) and after (T2) the rTMS sessions. We focused our statistical analysis on the analysis of variance (ANOVA) of measures repeated with three within-subjects and one between-subject factors. The within subjects factors were side (two levels, side more affected and side less affected), rTMS (two levels, before and after application of rTMS) and day (10 levels). The inter subject factor was group (two levels, real-rTMS and sham-rTMS). For the analysis of the gait task the factor side was not included. 


\section{Results}

Table 2 shows the mean and standard deviation at time T1 before the rTMS sessions, for each motor task in both groups. The CV of the reach task at the preferred speed with the less affected side was the only parameter significantly different between the groups $(t=4.218, p<0.05)$ in session T1. The motor UPDRS scores did not show significant differences between groups at $\mathrm{T} 1$. This shows that the groups were homogeneous in motor performance and clinical motor status. The UPDRS scores after DLPFC rTMS are summarized in the Fig. 1. There was no significant effect for either group.

Table 2. Values of the motor tasks in both groups before the sessions with rTMS

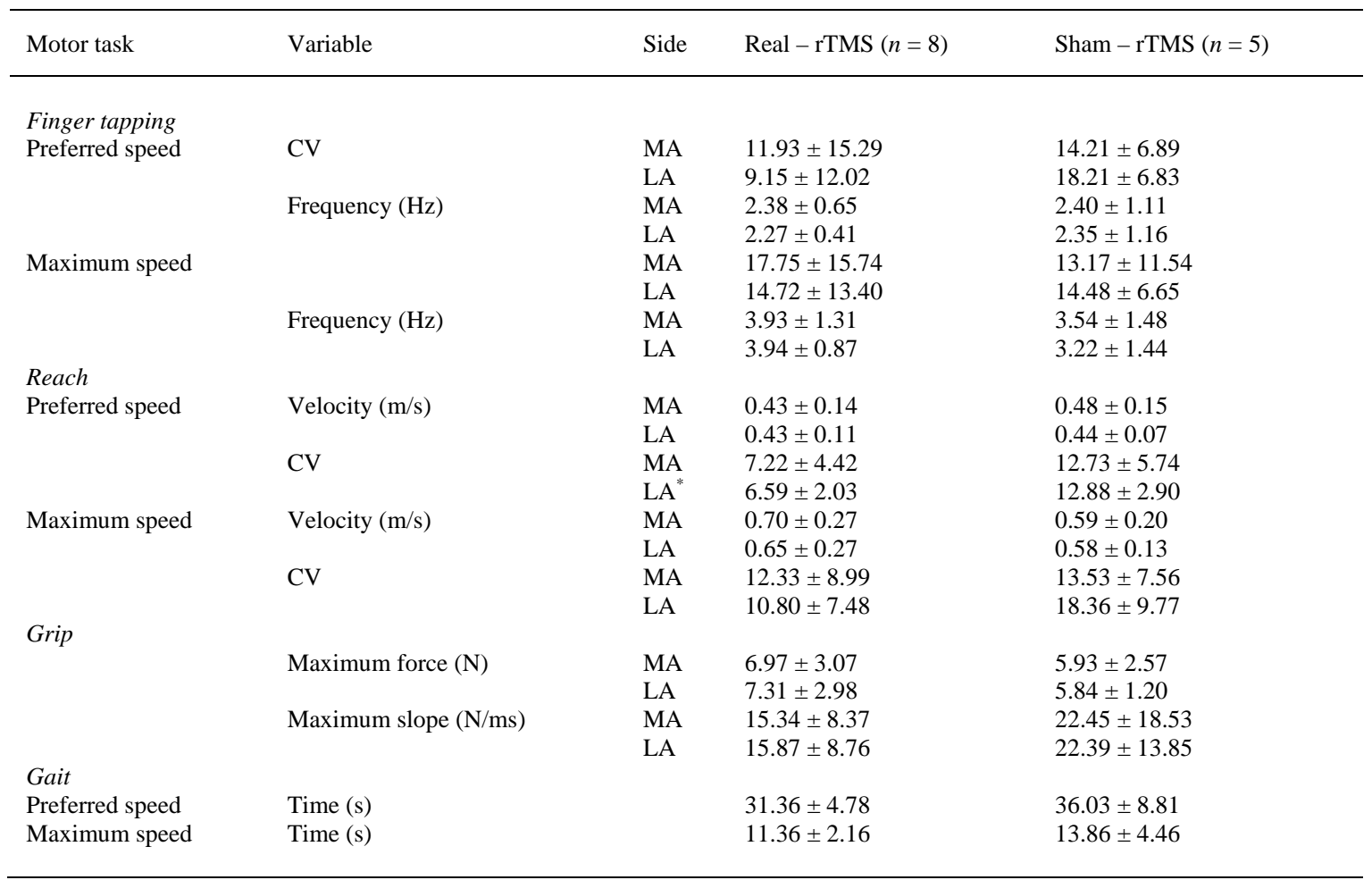

MA, more affected side. LA, less affected side. CV, coefficient of variation. ${ }^{*} \mathrm{p}<0.05$. Significant T1 values using t-test for independent samples.

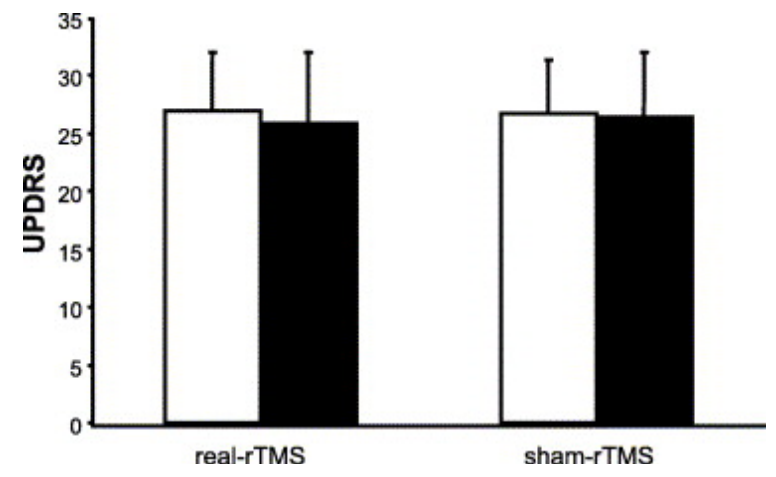

Fig. 1. Mean ( \pm SEM) values of the scores of the motor part of the Unified Parkinson's Disease Rating Scale in the real-rTMS and sham-rTMS groups before (white columns) and after (black columns) DLPFC 10-Hz repetitive transcranial magnetic stimulation. 
The ANOVA (side $\times r T M S \times$ day $\times$ group) showed the following results:

(i) The frequency of finger tapping at preferred speed and the CV at maximum speed showed a significant effect for the factor day $(F=13.027, p=0.005$ and $F=19.79, p=0.001)$ without significant interaction between the other factors ( Fig. 2). In the other parameters of the finger tapping there was no significant effect in the factors nor a significant interaction.
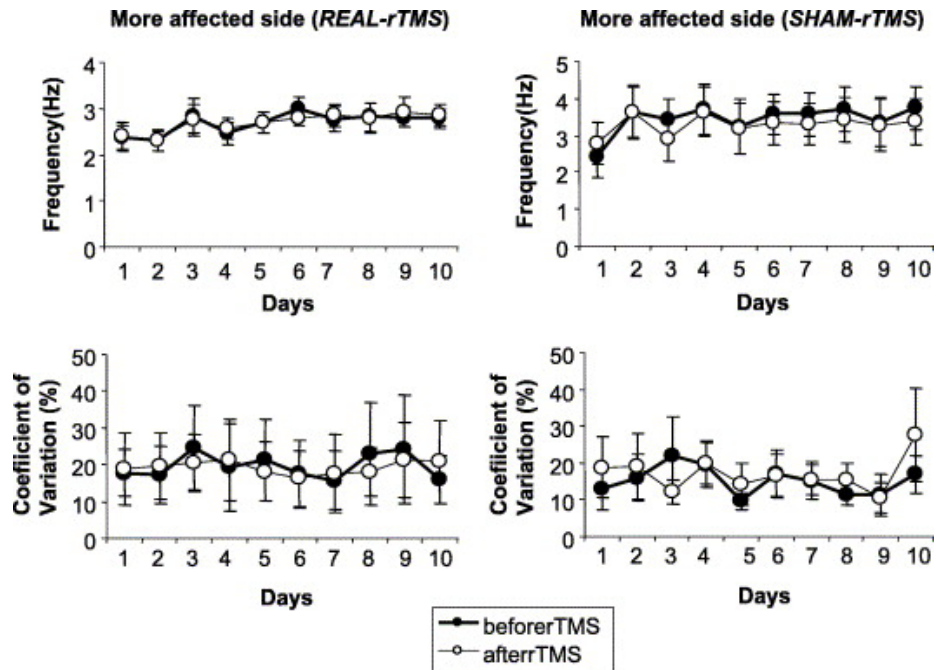

Fig. 2. Sequential assessment in the finger tapping test for the more affected side before and after DLPFC 10-Hz repetitive transcranial magnetic stimulation. Upper graphs show the frequency in the finger tapping test at preferred speed. Lower graphs show the coefficient of variation in the finger tapping at maximum speed. Left graphs correspond to real-rTMS group and right graphs to sham-rTMS group. The ANOVA shows a significant difference in the day factor $(p<0.05)$. Similar results were observed for the less affected side (not illustrated).

(ii) There was no significant difference on the maximum isometric force and the maximum slope in the grip task.

(iii) For the reach task at preferred speed only the factor day showed a significant effect for the velocity $(F=7.684, p=0.01)$ and $\mathrm{CV}(F=12.87, p=0.002)$ ( Fig. 3). Here was no significant effect for the reach task at maximum.
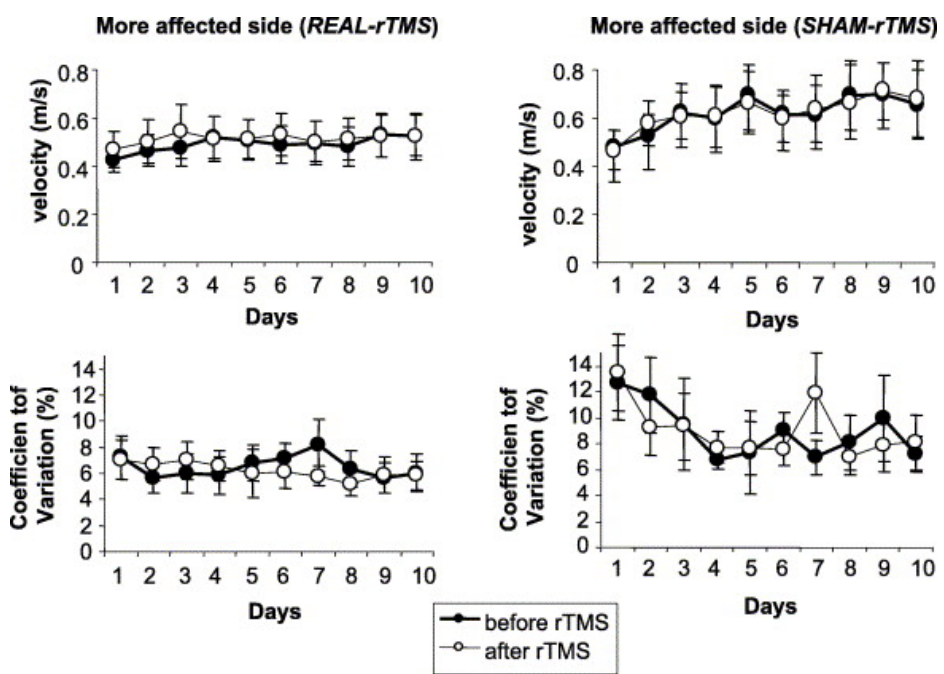

Fig. 3. Sequential assessment in the reaching task at preferred speed test for the more affected side before and after DLPFC 10-Hz repetitive transcranial magnetic stimulation. Upper graphs show the velocity and lower graphs the coefficient of variation. Left graphs corespondent to real-rTMS group and right graphs to sham-rTMS group. The ANOVA shows a significant difference in the day factor $(p<0.05)$. Similar results were observed for the less affected side (not illustrated). 
(iv) The gait at preferred and maximum velocity tasks showed a significant effect in the factor day $(F=8.47, p=0.007$ and $F=3.81, p=0.038$, respectively, Fig. 4$)$.

Gait at PREFERRED speed (REAL-rTMS)

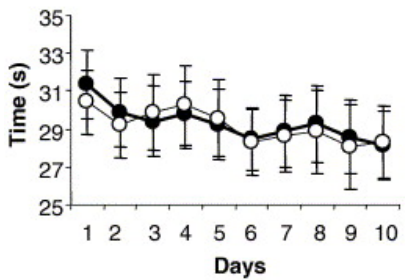

Gait at MAXIMUM speed (REAL-rTMS)

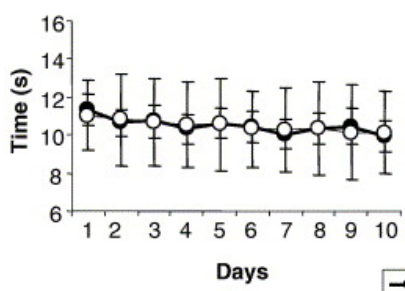

Gait at PREFERRED speed (SHAM-rTMS)

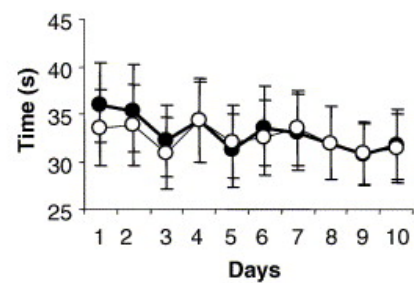

Gait at MAXIMUM speed (SHAM-rTMS)

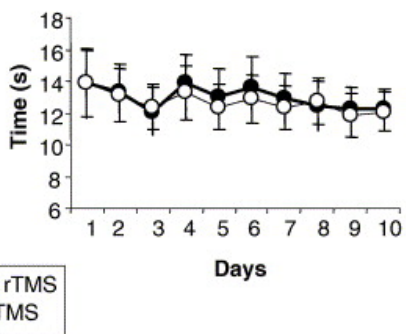

Fig. 4. Sequential assessment in gait at preferred speed (upper graphs) and maximum speed (lower graphs) before and after DLPFC $10-\mathrm{Hz}$ repetitive transcranial magnetic stimulation. Left graphs correspond to real-rTMS group and right graphs to sham-rTMS group. The ANOVA shows a significant difference in the day factor $(p<0.05)$.

\section{Discussion}

The present study shows that high frequency rTMS of the DLPFC over 10 days does not have a significant effect on the performance of motor tasks in PD patients and on the clinical motor status. The changes shown in this study can not be attributed to the effect of rTMS but rather to the possible effect of practice.

Ikeguchi et al. (2003) using $0.2 \mathrm{~Hz}$ in 6 successive sessions for 2 weeks showed a significant improvement in pronation-supination movements, buttoning up task and a increase in speed of walking over $10 \mathrm{~m}$. The authors attribute this improvement to the effect of TMS although Okabe et al. (2003) showed that this can be clearly attributed to a placebo effect. In our study, it is not possible to establish this cause-effect since the r-TMS had no significant effect over these tasks. It is possible that the changes in the motor performance observed in our study were due to practice effects (see below). Although other studies have reported changes in gait velocity and in finger tapping after rTMS (Khedr et al., 2003, Jäncke et al., 2004 and Lomarev et al., 2005), the protocol of stimulation used is different in the frequency, intensity, number of delivered stimuli, and the stimulated area. We have to note that the individual motor threshold was calculated before beginning the rTMS sessions. It is possible that the intensity used for rTMS was not exactly 90\% of RMT for each day, since Wassermann (2002) reported important variability in the RMT in normal subjects across different sessions. However, Lomarev et al. (2005) have not reported significant changes in the RMT in a group of 18 PD patients in a period of 4 weeks. Furthermore, intensity in the range of $80-115 \%$ of the RMT seems effective in affecting the prefrontal cortex (George et al., 1997 and Fregni et al., 2004). Thus, the possible variability of the individual RMT is not higher than the range cited. The number of delivered stimuli in our study was lower than that considered efficient in the treatment of depression in patients with PD (Fregni et al., 2004 and Boggio et al., 2005). However, Strafella et al. (2001) reported a release of endogenous dopamine in the caudate nucleus and Koch et al. (2003) showed an underestimation of time perception after application of 600 pulses at $1 \mathrm{~Hz}$ at $90 \%$ of RMT over the DLPFC. Thus, the number of delivered pulses in the present study would be sufficient to have an effect on the functionality of the DLPFC. Lomarev et al. (2005) found an improvement in the gait and in performance of complex hand movements but they stimulated four different cortical areas, therefore it is not possible to dissociate the role of the DLPC in this improvement.

We have included several aspects in the present study to test for a possible effect of rTMS: the presence of the sham-rTMS group, daily control session for a possible immediate effect of the rTMS and the performance of the upper limb motor tasks with both sides. To assess the actual effects of rTMS, 
placebo stimulation is a very important factor. A recent report showed that expectation of improvement releases dopamine in the caudate nucleus in PD (de la Fuente-Fernandez and Stoessl, 2002). Moreover, some authors suspect that the simple "off-head" stimulation could not be an effective "placebo", because patients may learn rapidly to distinguish between stimuli with and without a current flow (Boylan et al., 2001). Although we used the $45^{\circ}$ for the Sham-rTMS in order to avoid any stimulation effect, recent studies show that a $90^{\circ}$ position would be more adequate since the $45^{\circ}$ orientation of the coil can produce substantial stimulation of the cortex (Loo et al., 2000 and Lisanby et al., 2001). Thus, the absence of significant difference between the "active" and the "sham" procedures might be cause by a yet active placebo condition. Regarding motor performance, both groups were similar since only one parameter in one task was significantly different between them and this difference was constrained to the less affected side. The daily control of a possible immediate effect of the TMS was considered since other studies show a motor improvement and increase in corticospinal excitability after a single session of rTMS over the motor cortex of parkinsonian patients (Siebner et al., 2000 and Lefaucheur et al., 2004). All the subjects performed the motor tasks before and after the application of the TMS over 10 days, allowing rTMS effects to reach clinical level. However, the improvement in the motor tasks observed is most likely due to practice. In other studies patients with PD were able to improve the velocity of their movements after continued practice (Agostino et al., 1996). It is possible that the inclusion in this study of another real-rTMS group in which patients only performed the motor tasks on the first and the last day would help to determine the effect of motor practice, which could mask the real effect of the rTMS (Ghabra et al., 1999). However, although we carried out sessions at the same time of the day, considering the daily fluctuation of parkinsonian symptoms, the fluctuations in the motor performance over 10 days were evident. The reason we evaluated the effect of the rTMS in the hand contra and ipsilateral to the stimulated hemisphere was due to evidence that shows an influence of the rTMS over the motor cortex in the execution of movements in the ipsilateral hand (Kobayashi et al., 2004). This effect can be explained through the interhemispheric interaction between motor and premotor areas (Ferbert et al., 1992, Netz et al., 1995 and Hanajima et al., 2001). It would be of interest to know if this interhemispheric effect was present in the DLPFC. However, our results do not show a significant effect in the side factor nor in the rTMS factor and this issue can not be resolved.

In our study, the clinical assessment by UPDRS did not show any significant change after the DLPFC rTMS in both groups. However, regardless of the absence of motor and clinical improvement induced by rTMS, other different parameters could have been affected. Studies have reported that focal rTMS to the prefrontal cortex induced dopaminergic release in the caudate nucleus of healthy humans (Strafella et al., 2001) and changes in cerebral blood flow (Ikeguchi et al., 2003 and Rollnik et al., 2002). Although we believe that in our group the changes in motor performance were due to practice effect it is possible that the dopaminergic release reported by Strafella et al. (2001) could have occurred. It has been shown that there is no significant relation between improved clinical test and cerebral blood flow in the frontal areas after the application of rTMS (Ikeguchi et al., 2003). Moreover, changes in cortical excitability after rTMS do not have an affect on clinical parkinsonian symptoms or motor performance (Mir et al., 2005). Perhaps the use of other motor tasks specifically related to the DLPFC physiology (motor planning and response selection) would be more sensitive to changes of rTMS to this area (Goldman-Rakic, 1998). We evaluated several different motor tasks to explore if the possible effect of the rTMS was constrained to a specific movement. In fact all the tasks used in this studied are commonly used to measure the impaired motor performance in patients with Parkinson's disease. Higher temporal variability in the finger-tapping test in patients with PD has been previously demonstrated in various studies (Nakamura et al., 1978 and Ivry and Keele, 1989) as well as slower frequency of finger tapping at maximum velocity in comparison with elderly subjects (Shimoyama et al., 1990). Parkinson's disease affects the reaching movement kinematics (Tresilian et al., 1997) and reduced strength and ability to generate rapid contractions in isometric contractions have also been reported (Yanagawa et al., 1990 and Corcos et al., 1996). Overall it is well known that patients with PD show significantly slower velocity and difficulty in turning (Morris et al., 1994, Morris et al., 1996 and Mesure et al., 1999).

In summary, although in the last decade the studies of the therapeutic role of r-TMS in Parkinson disease have grown considerably, there are few studies of rTMS over the prefrontal dorsolateral cortex in patients with Parkinson's disease. Our results suggest that during 10 days of high frequency repetitive TMS over the DLPFC the patients only improved the performance of motor tasks due to practice and not due to the effect of rTMS, without affecting the clinical motor status.

\section{Acknowledgements}

Supported by a Xunta de Galicia (Spain) Grant. We wish to thank Noa Fogelson and Binith Cheeran for the revision of the manuscript. 


\section{References}

Agostino R, Berardelli A, Curra A, Manfredi M. The performance of rapid arm movements in Parkinson's disease. Adv Neurol 1996;69:135-46.

Boggio PS, Fregni F, Bermpohl F, Mansur CG, Rosa M, Rumi DO, et al. Effect of repetitive TMS and fluoxetine on cognitive function in patients with Parkinson's disease and concurrent depression. Mov Disord 2005;20:1178-84.

Boylan LS, Pullman SL, Lisanby SH, Spicknall KE, Sackeim HA. Repetitive transcranial magnetic stimulation to SMA worsens complex movements in Parkinson’s disease. Clin Neurophysiol 2001;112:259-64.

Chen R, Classen J, Gerloff C, Celnik P, Wassermann EM, Hallet M, et al. Depression of motor cortex excitability by low-frequency transcranial magnetic stimulation. Neurology 1997;48:1398-403.

Corcos DM, Chen C-M, Quinn NP, McAuley J, Rohwell JC. Strength in Parkinson's disease: relationship to rate of fore generation and clinical status. Ann Neurol 1996;39:79-88.

de la Fuente-Fernandez R, Stoessl AJ. The placebo effect in Parkinson's disease. Trends Neurosci 2002;25:302-6.

Ferbert A, Priori A, Rothwell JC, Day BL, Colebatch JG, Masden CD. Interhemispheric inhibition of the human motor cortex. J Physiol 1992;453:525-46.

Fox P, Ingham R, George MS, Mayberg H, Ingham J, Roby J, et al. Imagin human intra-cerebral conectivity by PET during TMS. NeuroReport 1997;8:2787-91.

Fregni F, Simon DK, Wu A. Pascual-Leone a non-invasive brain stimulation for Parkinson's disease: a systematic review and meta-analysis of the literature. J Neurol Neurosurg Psychiatry 2005;76:1614-23.

Fregni F, Santos CM, Myczkowski ML, Rigolino R, Gallucci-Neto J, Barbosa ER, et al. Repetitive transcranial magnetic stimulation is as effective as fluoxetine in the treatment of depression in patients with Parkinson's disease. J Neurol Neurosurg Psychiatry 2004;75:1171-4.

George MS, Wassermann EM, Kimbrell TA, Little JT, Williams WE, Danielson AL, et al. Mood improvement following daily left prefrontal repetitive transcranial magnetic stimulation in patients with depression: a placebocontrolled crossover trial. Am J Psychiatry 1997;154:1752-6.

Ghabra M, Hallet M, Wassermann EM. Simulataneous repetitive transcranial magnetic stimulation does not speed fine movement in PD. Neurology 1999;52:768-70.

Goldman-Rakic PS. The cortical dopamine system: role in memory and cognition. Adv Pharmacol 1998;42:707-11.

Hanajima R, Ugawa Y, Machii K, Mochizuki H, Terao Y, Enomoto H, et al. Interhemispheric facilitation of the hand motor area in humans. J Physiol 2001;531:849-59.

Ikeguchi M, Touge T, Nishiyama Y, Takeuchi H, Kuriyama S, Ohkawa M. Effects of successive repetitive transcranial magnetic stimulation on motor performances and brain perfusion in idiopathic Parkinson's disease. J Neurosci Sci 2003;209:41-6.

Ivry RB, Keele SW. Timing functions of the cerebelum. J Cogn Neurosci 1989;1:136-52.

Jäncke L, Steinmetz H, Benilow S, Ziemann U. Slowing faster finger movements of the dominant hand with lowfrequency rTMS of the hand area of the primary motor cortex. Exp Brain Res 2004;155:196-203.

Khedr EM, Farweez HM, Islam H. Therapeutic effect of repetitive transcranial magnetic stimulation on motor function in Parkinson's disease patients. Eur J Neurol 2003;10:567-72.

Khedr EM, Rothwell JC, Shawky OA, Ahmed MA, Hamdy A. Effect of daily repetitive transcranial magnetic stimulation on motor performance in PD. Mov Disord 2006; in press.

Kobayashi M, Hutchinson S, Theoret H, Schlaug G, Pascual-Leone A. Repetitive TMS of the motor cortex improves ipsilateral sequential simple finger movements. Neurology 2004;62:91-8.

Koch G, Oliveri M, Torriero S, Caltagirone C. Underestimation of time perception after repetitive transcranial magnetic stimulation. Neurology 2003;60:1844-6.

Lefaucheur J-P, Drouot X, Von Raison F, Menard-Lefaucheur I, Cesaro O, Nguyen J-P. Improvement of motor performance and modulation of cortical excitability by repetitive transcranial magnetic stimulation of the motor cortex in Parkinson's disease. Clin Neurophysiol 2004;115:2530-41.

Lisanby SH, Gutman D, Luber B, Schroeder C, Sackeim HA. Sham TMS: intracerebral measurement of the induced electrical field and the induction of motor-evoked potentials. Biol Psychiatry 2001;49:460-3.

Lomarev MP, Kanchana S, Bara-Jimenez W, Iyer M, Wassermann EM, Hallett M. Placebo-controlled study of rTMS for the treatment of Parkinson's disease. Mov Disord. 2005. Published Online: 6 Oct 2005; DOI: 10.1002/mds.20713.

Loo CK, Taylor JL, Gandevia SC, McDarmont BN, Mitchell PB, Sachdev PS. Transcranial magnetic stimulation (TMS) in controlled treatment studies: are some “sham”' forms active? Biol Psychiatry 2000;47:325-31.

Maeda F, Keenan JP, Tormos JM, Topka H, Pascual-Leone A. Modulation of corticospinal excitability by repetitive transcranial magnetic stimulation. Clin Neurophysiol 2000;111:1002-7.

Mally J, Farkas R, Tothfalusi L, Stone TW. Long-term follow-up study with repetitive transcranial magnetic stimulation (rTMS) in Parkinson’s disease. Brain Res Bull 2004;64:259-63.

Mesure S, Azulay JP, Pouget J, Amblard B. Strategies of segmental stabilization during gait in Parkinson's disease. Exp Brain Res 1999;129:573-81.

Mir P, Matsunaga K, Gilio F, Quinn NP, Siebner HR, Rothwell JC. Dopaminergic drugs restore facilitatory premotor-motor interactions in Parkinson's disease. Neurology 2005;64:1906-12.

Morris ME, Iansek R, Matyas TA, Summers JJ. The pathogenesis of gait hypokinesia in Parkinson's disease. Brain 1994;117:1169-81.

Morris ME, Iansek R, Matyas TA, Summers JJ. Stride length regulation in Parkinson's disease. Normalization strategies and underlying mechanisms. Brain 1996;119:551-68. 
Nakamura R, Nagasaki H, Narabayashi H. Disturbances of rhythm formation in patients with Parkinson's disease: Part 1. Characteristics of tapping response to the periodic signals. Percep Mot Skills 1978;46:63-75.

Netz J, Ziemann U, Homberg V. Hemispheric asymmetry of transcallosal inhibition in man. Exp Brain Res 1995;104:527-33.

Okabe S, Ugawa Y, Kanazawa I. Effectiveness of rTMS on Parkinson's Disease Study Group. 0.2-Hz repetitive transcranial magnetic stimulation has no add-on effects as compared to a realistic sham stimulation in Parkinson's disease. Mov Disord 2003;18:382-8.

Pascual-Leone A, Rubio B, Pallardo F, Catala MD. Rapid-rate transcranial magnetic stimulation of left dorsolateral prefrontal cortex in drug-resistant depression. Lancet 1996;348:233-7.

Pascual-Leone A, Tormos JM, Keenan J, Tarazona F, Canete C, Catala MD. Study and modulation of human cortical excitability with transcranial magnetic stimulation. J Clin Neurophysiol 1998;15:333-43.

Pascual-Leone A, Valls-Sole J, Wassermann EM, Hallett M. Responses to rapid-rate transcranial magnetic stimulation of the human motor cortex. Brain 1994a;117:847-58.

Pascual-Leone A, Valls-Sole J, Brasil-Neto JP, Cammarota A, Grafman J, Hallett M. Akinesia in Parkinson's disease: II. Effects of subthreshold repetitive transcranial motor cortex stimulation. Neurology 1994b;44:892-8.

Pascual-Leone A, Valls-Sole J, Brasil-Neto JP, Cohen LG, Hallett M. Akinesia in Parkinson's disease: I. Shortening of simple reaction time with focal, single-pulse transcranial magnetic stimulation. Neurology 1994c;44:884-91.

Paus T, Barret J. Transcranial magnetic stimulation (TMS) of the human frontal cortex: implications for repetitive TMS treatment of depresion. J Psychiatry Neurosci 2004;29:268-79.

Rollnik JD, Dusterhoft A, Dauper J, Kossev A, Weissenborn K, Dengler R. Decrease of middle cerebral artery blood flow velocity after low-frequency repetitive trasnscranial magnetic stimulation of the dorso-lateral prefrontal cortex. Clin Neurophysiol 2002;113:951-5.

Rossini PM, Barker AT, Berardelli A, Caramia MD, Caruso G, Cracco RQ, et al. Non-invasive electrical and magnetic stimulation of the brain, spinal cord and roots: basic principles and procedures for routine clinical application. Report of an IFCN Committee. Electroencephalogr Clin Neurophysiol 1994;91:79-92.

Schlaepfer TE, Rupp F. Effects of repetitive transcranial magnetic stimulation on inmediate early gene expression. In: Pascual-Leone A, Davey NJ, Rothwell J, Wassermann EM, Puri BK, editors. Handbook of transcranial magnetic stimulation. New York: Arnold; 2002. p.85-90.

Shimamoto H, Takasaki K, Shigemori M, Imaizumi T, Ayabe M, Shoji H. Therapeutic effect and mechanism of repetitive transcranial magnetic stimulation in Parkinson's disease. J Neurol 2001;248:48-52.

Shimoyama I, Ninchoji T, Uemura K. The finger-tapping test. A quantitative analysis. Arch Neurol 1990;47:681-4.

Siebner HR, Willoch F, Peller M, Auer C, Boecker H, Conrad B, et al. Imagin brain activation induced by long trains of repetitive transcranial magnetic stimulation. NeuroReport 1998;9:943-8.

Siebner HR, Rossmeier C, Mentschel C, Peinemann A, Conrad B. Short-term motor improvement after sub-thershold 5-Hz repetitive transcranial magnetic stimulation of the primary motor hand area in Parkinson's disease. J Neurosci Sci 2000;178:91-4.

Strafella AP, Pau T, Barrett J, Dagher A. Repetitive transcranial magnetic stimulation of the human prefrontal cortex induced dopamine release in the caudate nucleus. J Neurosci 2001;21:1-4.

Tergau F, Wassermann EM, Paulus W, Ziemann U. Lack of clinical improvement in patients with Parkinson's disease after low and high frequency repetitive transcranial stimulation. Electroencephalogr Clin Neurophysiol 1999;10:S281-8.

Touge T, Gerschlager W, Brown P, Rothwell JC. Are the after-effects of low frequency rTMS on motor cortex excitability due to changes in the efficacy of cortical synapses? Clin Neurophysiol 2001;112:2138-45.

Tresilian JR, Stelmach GE, Adler CH. Stability of reach-to-grasp movement patterns in Parkinson's disease. Brain 1997;120:2093-111.

Yanagawa S, Shindo M, Yanagisawa N. Muscular weakness in Parkinson's disease. In: Streifler MB, Korczyn AD, Melamed E, Youdim MBH, editors. Advances in Parkinson's disease. Parkinson’s disease: anatomy, pathology, and therapy, vol. 53. New York: Raven Press; 1990. p. 259-69

Wassermann EM. Variation in the response to transcranial magnetic brain stimulation in the general population. Clin Neurophysiol 2002;113:1165-71. 